\title{
BspKT6I, a new site-specific endonuclease which cleaves the GATC site producing two nucleotide 5'-protruding ends
}

\author{
Nina I.Shapovalova, Ludmila A.Zheleznaja ${ }^{1}$ and Nicholas I.Matvienko* \\ Institute of Protein Research and ${ }^{1}$ Institute of Theoretical and Applied Biophysics, Russian Academy \\ of Sciences, 142292 Pushchino, Moscow Region, Russia
}

Received October 29, 1993; Accepted November 5, 1993

\begin{abstract}
A new class II restriction endonuclease BspKT6I was isolated from soil thermophilic bacteria Bacillus species KT6. The enzyme was purified by chromatography on blue-agarose, heparineSepharose and hydroxylapatite. Digestion of the T7 DNA has revealed that the enzyme is an isomer of Sau3AI. Cleavage points were determined by the primed-synthesis reaction (1) using the M13mp18 DNA. The cleavage product resulted in the band comigrated with G (Fig. 1). Upon addition of the DNApolymerase, a band appeared two nucleotides higher. These results indicate that the enzyme recognizes the palindromic sequence GlATC and cleaves it as indicated by the arrow creating two nucleotide $5^{\prime}$-extensions. It is the first isomer of Sau3AI producing such ends. The DNA fragments produced by this enzyme can be ligated with $P v u I$-produced DNA fragments. The $B s p$ KT6I is sensitive to dam-methylation. It does not cleave the pBR322 isolated from the E.coli dam ${ }^{+}$strain and results in almost complete digestion of pBR322 isolated from the dam ${ }^{-}$ strain (Fig. 2). The optimal reaction buffer is $10 \mathrm{mM}$ Tris $-\mathrm{HCl}$ (pH 7.5), $10 \mathrm{mM} \mathrm{MgCl}_{2}$.
\end{abstract}

\section{REFERENCE}

1. Brown,N.L. and Smith,M. (1980) Methods Enzymol. 65, 391-404.

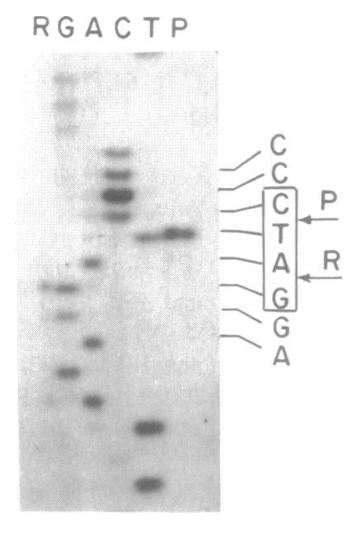

Figure 1. Determination of the BspKT6 cleavage site. R: The template of primedsynthesis reaction was cleaved with the BspKT6I; P: The product from line R was treated with DNA-polymerase; G, A, C, T: The sequence ladder through the BspKT6I recognition sequence, using termination method.

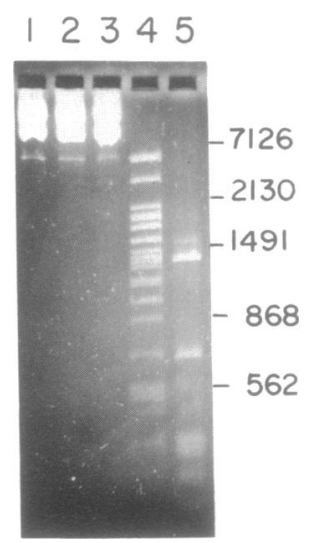

Figure 2. Sensitivity of the BspKT6I to dam-methylation of DNA. (1) pBR322 from $E$. coli dam ${ }^{+}$; (2) pBR322 from E. coli dam; (3) pBR322 from E. coli dam ${ }^{+}$ treated with the BspKT6I; (4) Molecular length markers in bp: T7 DNA treated with the Bli 736I; (5) pBR322 from E.coli dam treated with the BspKT6I. 